more abundant of the E.coli tRNAs). The processing of the transcript of the complete unit releases four RNA species, in the order $16 \mathrm{~S}$, tRNA, 23S, 5S RNA; the enzyme that appears to undertake this reaction is ribonuclease III and by showing that a deletion phage lacking the end of the $16 \mathrm{~S}$ sequence can still generate $23 \mathrm{~S}$ and tRNA sequences, Yamamoto et al. (op. cit.) were able to infer that the complete precursor sequence is not required for proper processing. This supports the idea that processing can occur before transcription has been completed.

\section{Nuclear charge distributions}

\section{from P. E. Hodgson}

NEw measurements of the cross section for the elastic scattering of electrons by lead have recently cleared up an outstanding discrepancy concerning the charge distribution in the interior of the nucleus. It has for a long time been uncertain whether there is a dip or a hump in the middle of ${ }^{208} \mathrm{~Pb}$, and it has now been established that there is a small hump.

At first sight this result seems surprising. We know that the protons in the nucleus repel each other by their electrostatic charges, and this might lead us to expect a dip in the charge distribution in the middle of the nucleus. We must however think of the protons as occupying shell model orbits, and the total charge distribution is found by adding the squared moduli of the wavefunctions of the protons in all the occupied orbits. Now the wavefunctions of the protons in S-orbits, with zero orbital angular momentum, have a finite value at the centre of the nucleus, whereas the wavefunctions of protons in all other orbits are zero at the centre. The value of the charge density at the centre of the nucleus thus depends quite critically on the number of protons in S-orbits. In lead the protons in the $3 \mathrm{~S}$ orbits have the smallest binding energy, and so we might expect that these form a hump at the centre of the charge distribution, and this hump is indeed given by most calculations of the charge distribution of lead, for example those made using the Hartree-Fock theory.

Until recently, however, the experimental determinations of the charge distribution have tended to give a small central dip, and this could not be reconciled with the theoretical result. There was however some uncertainty about this because of the difficulty of obtain- ing the charge distribution from the measurements of electron scattering.

Electrons only interact with the nucleus throughout their electromagnetic field, so if we scatter electrons by nuclei we should learn about the charge distribution of the nucleus. Unfortunately, although it is possible to calculate the scattering from a known charge distribution, it is not easy to reverse the calculation and obtain the charge distribution from the electron elastic scattering cross section. Some quite sophisticated techniques have been developed to do this, and the differences between them account for much of the uncertainty about the form of the charge distribution in the centre of the nucleus.

The simplest method is to assume a suitable analytic form for the charge distribution and then vary its parameters to optimise the fit to the electron elastic scattering cross section. One of the most popular forms is the Fermi function $[1+\exp \{(r-R) / d\}]^{-1}$ which is uniform in the interior and falls rapidly to zero around the radial distance $R$, the steepness of fall depending on the parameter $d$. This was soon found to be insufficiently flexible, so it was multiplied by various polynomials such as $\left(1+w r^{2}\right)$, which makes it possible to introduce dips and humps in the interior.

Many analyses were made in this way, and it was often possible to obtain excellent fits to the scattering cross sections, but there was some doubt about the interpretation of the resulting charge distributions. It was realised that the scattering is most sensitive to the charge distribution in the 'knee' region at the surface of the nucleus, and the parameters are largely determined by the behaviour of the assumed charge distribution in this region, so that it is dangerous to assume that this gives us reliable information about the charge distribution in other regions such as the centre of the nucleus.

Much effort has therefore been devoted to the development of the socalled model-independent ways of determining the charge distribution from the scattering cross sections. Some of these use a series of concentric shells of charge, and adjust the densities of each, while others express the charge distribution as the sum of a series of terms each consisting of a suitable function of the radial distance, with different ranges and coefficients. Some important work of this type has been done by Friedrich and Lenz ( Nucl. Phys., A183, 532; 1972) and Friar and Nigele (Nucl. Phys., A212, 93; 1973), so that it is now possible to obtain the charge distribution without making any unduly restrictive assumptions about the form of the distribution.

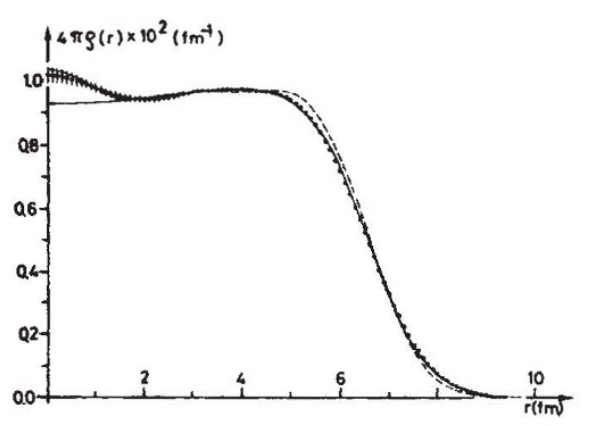

The charge distribution of lead, as determined experimentally and by Hartree-Fock calculations. The experimental points correspond to the analysis of the Mainz data, the dashed line to the calculations of Vautherin and Brink using the Skyrme force, and the full line the result of an analysis using a three-parameter Fermi distribution that does not give an acceptable fit to the electron scattering data.

The new measurements that have recently been made to resolve the difficulty about the charge distribution in the centre of lead are due to the group of Professor Ehrenberg at the University of Mainz (Euteneur, Friedrich and Voegler, Phys. Rev. Lett., 36, 129; 1976). They scattered electrons of 120 , 200 and $290 \mathrm{MeV}$ from lead, and analysed the results using a very fiexible expansion of the charge distribution in terms of a series of spherical Bessel functions of various ranges. Their results, as shown in the figure, provide definite evidence in favour of a hump in the centre of the nucleus, in accord with the theoretical calculations.

This work shows how accurately we now know the charge distribution of some nuclei. There still remain some uncertainties, especially in the region of low densities at the edge of the nucleus, and this will doubtless be the subject of further studies using electrons of higher energies.

\section{Role for histone H1 in chromatin condensation?}

\section{from Tom Barrett}

THE lysine-rich histone $\mathrm{H} 1$ is the most unusual of the five major histone species found in eukaryotic chromatin. In contrast to the other histones which show a strict sequence conservation over a very long evolutionary period, the amino acid sequence of histone $\mathrm{H} 1$ varies to some extent (Rall and Cole, J. biol. Chem., 246, 7175; 1971). Its molecular weight (about 23,000 daltons) is greater than that of the other major histone species $\mathrm{H} 2 \mathrm{~A}, \mathrm{H} 2 \mathrm{~B}, \mathrm{H} 3$ and $\mathrm{H} 4$. It contains a very high proportion of 